\title{
Application of the BP Neural Network Model of Gray Relational Analysis in Economic Management
}

\author{
Zhuojie Li 1 ) \\ Department of Economics and Management, Zhengzhou University of Light Industry, Zhengzhou 450002, China \\ Correspondence should be addressed to Zhuojie Li; lizhuojie@zzuli.edu.cn
}

Received 26 November 2021; Accepted 8 January 2022; Published 17 February 2022

Academic Editor: Miaochao Chen

Copyright $\odot 2022$ Zhuojie Li. This is an open access article distributed under the Creative Commons Attribution License, which permits unrestricted use, distribution, and reproduction in any medium, provided the original work is properly cited.

\begin{abstract}
According to the BP neural network algorithm, for the problem that the input variables of the BP network cannot be automatically optimized during the modeling process of the multivariable complex system, it is used to establish a neural network optimization algorithm according to the gray correlation analysis (GM2 BPANN). Using the data of China's grain production forecast, the stepwise regression method and the gray GM $(1, N)$ model method were compared and tested. The results show that the new model can comprehensively and extensively process a large number of input variables by using the concept of the gray correlation degree, without having to go through special subjective screening and hence improving the adaptability of the BP network, and at the same time, it can obtain better prediction accuracy and stability. Through the empirical test, the prediction ability of the three methods of regression, GM $(1, N)$ gray system, and GM-BPANN model is compared. It is proved that the GM-BPANN optimization algorithm that combines the gray relational analysis and BP neural network method can enhance the multivariable processing ability and network adaptability of the BP network and has good prediction accuracy and stability.
\end{abstract}

\section{Introduction}

The prediction problem, in essence, is the process of obtaining the output of the future result under the action of the prediction operator by taking the known situation in the past as the input. During the last ten years, with the development of intelligent technology, new technologies and new methods such as gray system theory prediction technology, prediction technology combined with the expert system, fuzzy inference prediction technology, and artificial neural network prediction technology have frequently appeared, which have solved many of the traditional methods. Defects have been widely used [1-8].

The artificial neural network is a mathematical method that does not need to determine the mutual mapping relationship between input and output in advance. Generally speaking, a deep neural network model can constitute a deep learning model. The network structure of deep learning models is generally more complex, and the number of parameters is often relatively large. Therefore, in order to make the model have better performance, people generally rely on the corresponding manually labeled data to do the corresponding training; otherwise, there may be overfitting problems or training difficulties. However, generally in actual situations, it is difficult to obtain enough data. Therefore, data in some application fields are still seriously missing. For example, in the world, the amount of rare ores is small, and the corresponding data are often difficult to collect. Another example is as follows: it is relatively difficult for people to collect some relevant data on endangered precious animals or plants. In addition, even if sufficient data are available, the cost of manual labeling during this period is often high. Therefore, it is difficult for people to use enough data to train the corresponding neural network model enough, which will seriously affect its performance, which may lead to a serious overfitting problem of the model. Therefore, using less data to maximize the performance of the model is a hotspot in the field of deep learning research. Another issue worthy of attention in this field is that eventhough deep neural networks have completed simulation training based on a large number of data samples, they still cannot apply the simulation results to other fields. For 
example, the Go robot AlphaGo developed by Google has defeated Go masters from various countries in many competitions, but it appears to be powerless in the game of checkers. This is equivalent to that artificial intelligence which consumes a lot of manpower, material, and financial resources to engage in simulation training, but it is difficult to apply in cross-industry or just cross-project activities. With the improvement of AI technology, people hope that artificial intelligence technology can achieve capabilities comparable to those of natural people. Artificial intelligence only needs to observe a small number of samples to train and simulate new things. For example, a human child has never seen a panda, but he often only needs to observe the corresponding photos a few times to distinguish it when he sees a panda next time. This is mainly due to the fact that humans can often use experience and knowledge. At present, deep learning lacks the ability of humans to learn new things quickly. Therefore, with the aim of solving the problem of deep learning model's serious dependence on the amount of data, people gradually began to focus on small sample learning technology. At present, people's analysis of corresponding small sample learning is often based on image classification and target detection. The research content of this study is mainly focused on the improvement of the technical scheme of small sample learning in image recognition tasks [9-15].

Domestic research in this area is not lagging behind. More representative scholars have paid attention on the application of neural networks and genetic algorithms in the field of water science and established BP neural network models for rainstorm, flood, and weather forecasting; some scholars have also established analysis of the BP neural network prediction model for debris flow activity; in addition, some people use the neural network to solve the identification problem of the structural damage model in mechanical processing. In recent years, in view of the inherent shortcomings of the BP network that cannot automatically optimize multiple variables, the learning process converges slowly and is easy to fall into local minima; many scholars at home and abroad use rough sets and genetic algorithms in combination with them to achieve the purpose of optimization. The improvement of it is also continuous. For example, Amit Gupta and Siuwa M. Lam established a BP network with decreasing weight for noisy data; Yutaka Fukuoka et al. established a modified BP network to avoid falling into local minima [16-20].

As the core of the subject of artificial intelligence, neural networks are the basis for making computers intelligent. They are interoperable and inclusive and can organically integrate multiple types of theories. The main content is to use algorithms to enable the machine to have a certain simulation ability, to independently complete some behaviors that only humans can do before, to establish the machine's own knowledge structure and skill system, and to update and improve its knowledge structure in real time. As one of the most important technologies of artificial intelligence, it is the basis for the realization of mechanical intelligence. Generally speaking, neural networks are mainly used to summarize and integrate information, which is then deduced by artificial intelligence [21-25].

Gray system theory is also a forecasting method which has been commonly utilized in these years. Since Professor Deng Julong proposed the gray system theory in 1982; many experts and scholars have devoted themselves to it and continued to expand his research results. The more representative ones are Liu Sifeng, Dang Yaoguo, Guo Tianbang, and others from Huazhong University of Science and Technology, who successively conquered the generalized gray correlation. Gray parameter linear programming (LPGP) drift and positioning solution, gray econometric model, gray production function model, and other research fields, in these years, new progress has gained more and more attentions in the research of the gray model optimization and gray game model. In terms of application, gray system theory is successfully applied in many fields, such as engineering control, economic management, social systems, ecosystems, and other fields. In addition, it also can be used in complex and changeable agricultural systems, for example, in water conservancy. Agricultural economy and other aspects have also made gratifying achievements. For example, Su Bianping and Cao Yanping used the gray system theory to eliminate the noise pollution in the independent variable observation data, improved the traditional multiple linear regression analysis method, and established a gray multiple linear regression analysis model to analyze the employment problem in Shaanxi Province; Li Chunsheng used the gray system theory and methods, the gray forecast and control analysis of grain production in Hubei Province, and the establishment of a gray forecast, control and management GM $(1, N)$ model of grain production; Guo Jianqiu and others used the principles and methods of gray correlation analysis to analyze the performance of 15 soybean varieties The degree of influence of main traits is on yield. Dang Yaoguo and Liu Sifeng used a combination of qualitative analysis, gray system theory, and linear regression to determine the constraints of the linear programming model for the adjustment of the internal industrial structure of my country's primary industry and established the linear programming mathematics for the adjustment of the internal structure of my country's primary industry [26-30].

The degree of correlation between them is considered to be large; whereas, the degree of correlation between them is small. The correlation degree analysis of gray system theory is different from the correlation analysis of mathematical statistics theory, the difference lies in the first, and their theoretical basis is different. Correlation degree analysis is based on the gray process of the gray system, while correlation analysis is based on the stochastic process of probability theory. Correlation analysis is to compare the time series of factors, and correlation analysis is to compare the array of factors. Correlation analysis does not require too much data, while correlation analysis requires enough data. Fourth, the emphasis of research is different. The correlation analysis mainly studies the dynamic process, while the correlation analysis mainly studies the static process. Therefore, the analysis of correlation degree is more 
adaptable, and its application in the social economic system is more original [31-33].

The successful application of the BP neural network (Figure 1) and gray system theory in many scientific fields has won the attention and affirmation of the international and domestic academic circles, but there are still few attempts to combine gray system theory with neural network prediction algorithms. In this study, based on the BP neural network algorithm, try to combine it with the gray system correlation analysis method to solve the optimization selection problem of the BP neural network input variables and establish the GM 2 BPANN optimization model. This new method could process a great number of input variables through the calculation of the gray correlation degree. It can be used to select variables as comprehensive and extensive as possible, without special subjective screening, and hence improve the adaptability of the BP network and making it more effective. It is applied to the modeling of multivariable complex systems. Then, the GM 2 BPANN optimization model was applied to my country's grain output prediction and compared with the stepwise regression and GM $(1, N)$ model methods, and the detection samples were used to make the year-by-year prediction to test the prediction effect. Through the calculation of the gray correlation degree, a large number of input variables can be processed comprehensively and widely without special subjective screening, thus enhancing the adaptability of the BP network, and at the same time, it can obtain better prediction accuracy and stability.

\section{Overview of Gray System Modeling Methods}

The neutral network only needs to train itself to achieve the purpose of learning specific rules, so that it can obtain the best value when the input value is given. The effect is close to the desired output value. Gray system theory is a new field of control theory. It is the product of the application of cybernetic viewpoints and methods to the social economic system, as well as the product of the combination of control theory and operations research. It takes the gray system as the research object, takes the whitening, desalination, quantification, modeling, and optimization of the gray system as the core, and aims to predict and control the development of various gray systems. Its main research content includes gray system modeling theory, gray factor correlation analysis theory, gray prediction theory and decision theory, gray system analysis and control theory, and gray system optimization theory. In 1981, Chinese cybernetics expert Professor Deng Julong was the first person to use the theory of gray system, then wrote many publications and other intellectual property on it, and established gray system theory. Since 1982, gray system theory has been applied in agriculture, industry, and meteorology. Other fields have been successfully applied. It can solve problems in special fields with unknown factors. It is widely used in agriculture, geology, meteorology, and other disciplines.

The most characteristic of gray situation decisionmaking is that it is suitable to deal with the decision-making problem which contains gray elements in the data, i.e., incomplete information. In the regional development

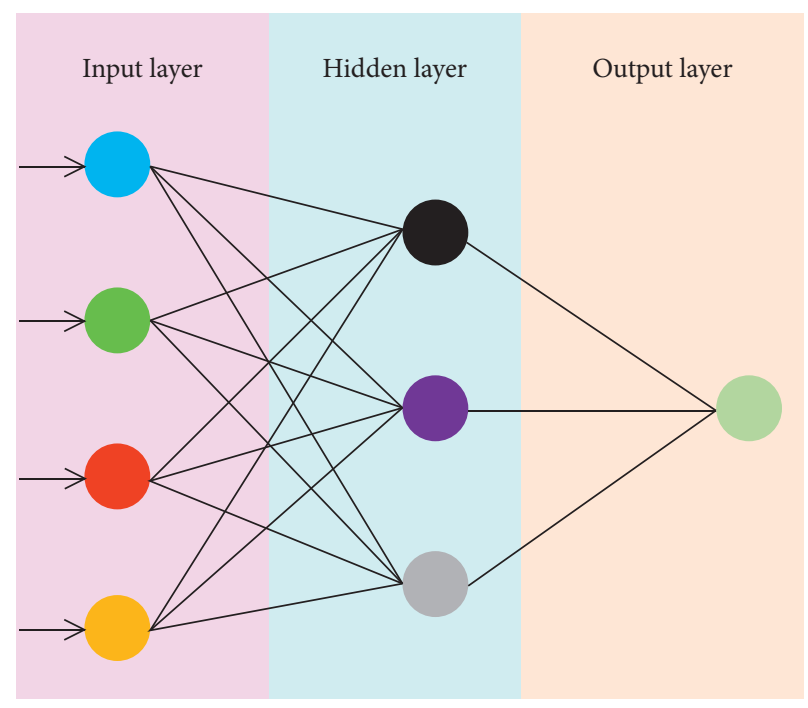

FIgURE 1: BP neural network.

activities, many problems are solved by decision-making under the condition of incomplete information. Therefore, the gray situation decision-making is one of the decisionmaking analysis methods commonly used in the study. Decision-making, in general, consists of the following four basic elements: (1) the event, that is, the thing to be dealt with; (2) the countermeasure, that is, the measure to be taken to deal with something; (3) the effect, that is, the effect of using a countermeasure to deal with an event; and (4) the objective, that is, the criteria used to evaluate the effect. The so-called decision-making is, for a (or some) event, considering a number of countermeasures to deal with, different countermeasures effect is different, and then with some (or some) goals to measure, from these countermeasures to choose (or a group) the best effect. Gray situation decisionmaking is a kind of the decision-making analysis method which considers the four factors of decision-making, such as event, countermeasure, effect, and goal. The mathematical model of gray situation decision-making is essentially a comprehensive description of the relationship among the four elements of decision-making by using relevant mathematical language. This description includes the following aspects of the basic.

The object of gray system theory investigation and research is the system with incomplete information. The unknown domain can be studied and predicted by the known information to achieve the purpose of understanding the whole system. It can effectively overcome the contradiction of insufficient data or short system cycle. Gray forecasting is an important aspect of gray system application. There are three commonly used gray prediction models: GM $(1,1)$, GM $(2,1)$, and GM $(1, N)$. In this study, the GM $(1, N)$ model is used as a comparative test model for calculation and analysis.

2.1. Principles of Gray Relational Analysis. The degree of relevance is a measure of the relevance of two sequences. The correlation analysis method of gray system theory is the basis 
of gray system analysis, prediction, and decision-making. It can reveal the characteristics and degree of the dynamic relationship of things, has the characteristics of less sample size, no need for typical distribution rules, and less calculation, and there will be no inconsistency between quantitative results and qualitative analysis. The basic principles and calculation steps are as follows.

If $m$ time series groups are

$$
\left\{X_{1}^{(0)}(t)\right\},\left\{X_{2}^{(0)}(t)\right\}, \ldots,\left\{X_{m}^{(0)}(t)\right\}, \quad(t=1,2, \ldots, N) .
$$

Set another time series are

$$
\left\{X_{0}^{(0)}(t)\right\}, \quad(t=1,2, \ldots, N) .
$$

The gray correlation analysis of the former and latter generally includes the following calculations and steps:

(1) Standardized transformation of original data

(2) Calculate the correlation coefficient. The correlation coefficient $L_{0 i}(k)$ between the input sequence $\left\{X_{0}(k)\right\}$ and the output sequence $\left\{X_{i}(k)\right\}$ at time $t=k$ can be calculated by the following formula:

$$
L_{0 i}(k)=\frac{\Delta_{\min }+\Delta_{\max }}{\Delta_{0 i}(k)+\rho \Delta_{\max }} .
$$

In the formula, $\Delta_{\max }$ and $\Delta_{\min }$, respectively, represent the maximum and minimum values of the absolute difference at each time of all comparison sequences, and $\rho$ is the resolution coefficient.

(3) Relevance:

$$
r_{0 i}=\frac{1}{N} \sum_{k=1}^{N} L_{0 i}(k),
$$

where $r_{0 i}$ is the degree of relevance between the input sequence and the output sequence, and $N$ is the length of the comparison sequence. Generally speaking, the degree of association also satisfies the three axioms of equivalent "relationship," namely, reflexivity: $r_{00}=1$; symmetry: $r_{0 i}=r_{i 0}$; and transitivity: if

$$
\begin{aligned}
& r_{0 a}>r_{0 b}, \\
& r_{0 b}>r_{0 c},
\end{aligned}
$$

then

$$
r_{0 a}>r_{0 c}
$$

(4) Sort the order of associations. At present, there are two representative association sequences: "order" and "partial order." Generally speaking, as long as each factor can form a relationship and calculate the degree of relevance, it will always be "ordered." Only when there is no "reference point" or no reference mother sequence, can the phenomenon of "partial order" appear.
(5) List the incidence matrix. If there are $n$ parent sequences,

$$
\left\{Y_{1}\right\},\left\{Y_{2}\right\}, \ldots,\left\{Y_{n}\right\}, \quad(n \neq 2),
$$

and $m$ subsequences

$$
\left\{X_{1}\right\},\left\{X_{2}\right\}, \ldots,\left\{X_{m}\right\}, \quad(m \neq 1),
$$

each subsequence $\left\{Y_{i}\right\}$ has a degree of relevance $\left[r_{i 1}, r_{i 2}, \ldots, r_{i m}\right]$ to the parent sequence $r_{i j}(i=1,2, \ldots, n ; j=1,2, \ldots, m)$ and will be arranged appropriately to obtain the relevance matrix. The relevance matrix can not only be used as the basis of advantage analysis but also can be used as decision-making in accordance.

2.2. Gray GM $(1, N)$ Model Principle. As a kind of the intelligent information processing system, the artificial neural network is the core part to realize its function. The BP neural network is a multilayer feedforward network trained according to error back propagation (error back propagation for short). The GM $(1, N)$ model is a first-order linear dynamic model that describes multiple (multivariate) variables. It is mainly used for system dynamic analysis and can analyze multivariate complex systems. The basic steps and principles of establishing the GM $(1, N)$ model are as follows.

For the original data matrix $X_{N}^{(0)}$ with $N$ number sequences and the number sequence length $M$.

Step 1: calculate the accumulated data matrix $X_{N}^{(1)}$ once and then calculate the accumulated difference data sequence $\left\{\alpha^{(1)}\left(x_{1}^{(1)}, i\right)\right\}$ of $x_{1}^{(1)}$;

Step 2: construct the matrix $X(A, B)=B$;

Step 3: solve the gray parameters with the least square method $\widehat{a}=\left(B^{T}, B\right)^{-1} B^{T} Y_{M}$;

Step 4: substitute the gray parameters into the time function:

$$
\begin{aligned}
x^{(\hat{1})}(t+1)= & {\left[x_{1}^{(0)}(1)-\sum_{i=2}^{N} \frac{b_{i-1}}{a} x_{i}^{(1)}(t+1)\right] e^{-a t} } \\
& +\sum_{i=2}^{N} \frac{b_{i-1}}{a} x_{i}^{(1)}(t+1) .
\end{aligned}
$$

Step 5: substituting the gray parameters into the time function, after calculating the calculated value of the generated data sequence $x^{(\widehat{1})}(t+1)$, and then deriving the derivative of $x^{(\widehat{1})}$ to obtain $x^{(\widehat{0})}(t)$ and calculating the difference $\varepsilon^{(0)}(t)$ between $x^{(\widehat{0})}(t)$ and $x^{(0)}(t)$ and the relative error $e(t)$.

Step 6: while establishing the model, the GM $(1, N)$ model parameters are Laplace transformed, and the transfer function $w_{i}(s)$ of the $i^{\text {th }}$ influencing factor to the dynamic link of the target is given under the zero initial condition. 


\section{The Principle and Method of the BP Neural Network}

The corresponding algorithm is called the $\mathrm{BP}$ algorithm. The method used is the gradient descent method, which is to use gradient search technology, in order to minimize the error mean square error between the actual output value of the network and the expected output value as the final goal. The neural network is the most common type in the field of the ANN, as shown in Figure 2. Because the neural network requires a lot of data for simulation training in the initial stage and requires high hardware equipment of the computer itself, it is often difficult to pass training, way to get a relatively good performance network. However, in recent years, with the continuous advancement of GPUs and corresponding labeled data, the CNN has shown better and better results in dealing with image recognition or image classification problems. It is precisely because of this advantage of the $\mathrm{CNN}$ that it is widely used in face recognition, object recognition, and other occasions. In recent years, the successful application of convolutional neural networks in image recognition has received widespread attention. Generally speaking, common image recognition methods can generally be divided into the following three types: decision theory recognition, syntactic pattern recognition, and fuzzy pattern recognition. Among them, a major feature of syntactic pattern recognition is the use of several structural features to form a single recognized object, which can accurately describe the characteristics of the image. Suppose a picture is composed of lines, curves, and polylines, according to specific conventions. Therefore, it is often combined with the knowledge of statistical decisionmaking in mathematical statistics to reconstruct the secondary space to achieve the purpose of image recognition. Commonly used methods include the similar judgment method, similarity analysis method, and function classification method. Among them, fuzzy pattern recognition is mainly through training to imitate the process of identifying things in the human brain, which can not only obtain more accurate classification of objective things but also improve the simplicity of the recognition module. Therefore, it is also a method for the above two recognition methods, a kind of supplement.

As an important artificial intelligence technology, the neural network has also been widely studied and applied in the field of prediction. It has a high degree of nonlinearity and excellent adaptive learning capability, which helps to be commonly used in various fields, such as function approximation, pattern recognition, data compression, and so on. The guiding ideology of the BP network learning rules can be expressed as the mathematical expression, as is shown in the following formula.

$$
x_{k+1}=x_{k}-a_{k} g_{k},
$$

where $x_{k}$ is the current weight and threshold matrix, $g_{k}$ is the gradient of the current performance function, and $a_{k}$ is the learning rate.

The basic principles of the proposed three-layer BP neural network model discussed in this study are briefly described as follows.
For two discrete time series of $N$ sample sets $\left\{\left(x_{k}, y_{k}\right) \mid x \in R^{m}, y \in R^{n}\right\},(k=1,2, \ldots, N)$, the total sample is divided into training samples $\phi_{1}$ and testing samples $\phi_{2}$.

The most widely used is back propagation (BP). Since its establishment, related research studies and application results are emerging one after another, involving many fields such as the engineering control, economic management, social system, and ecological system. First, use the training sample to establish the mapping relationship after training, and then, use the test sample to test whether the network can give the correct input and output relationship. A three-layer BP neural network with input node number $m$, output node number $n$, and hidden node number $p$ is adopted. The activation function from the input layer to the hidden layer adopts the sigmoid type, and the activation function from the hidden layer to the output layer adopts a linear function. The relationship between the input and output of the available network is as follows:

$$
\hat{y}_{k}(t)=\sum_{j=1}^{p} v_{j k} f\left[\sum_{i=1}^{m} w_{i j} x_{i}(t)+\theta_{j}\right]+r_{k},
$$

where $f(x)=\left(1 / 1+e^{-x}\right), k=1,2, \ldots, n, t=1,2, \ldots, N_{1}, x_{i}$ is the input of the network, $y_{k}(t)$ is the expected output of the network, $\hat{y}_{t}$ is the actual output, $w_{i j}$ is the network weight between input node and hidden layer node, $v_{i j}$ is the network weight between hidden layer nodes and output nodes, $\theta_{j}$ is the threshold of hidden nodes, and $r_{k}$ is the threshold at the output node. Set the total error of the network to be less than $\varepsilon_{1}$; then, the output node error is

$$
E_{1}=\frac{1}{2} \sum_{k=1}^{N_{1}} \sum_{t=1}^{n}\left[y_{k}(t)-\hat{y}_{k}(t)\right]^{2} \leq \varepsilon_{1} \text {. }
$$

Assuming that the average mean square error of the test sample is less than $\varepsilon_{2}$, then there is

$$
E_{2}=\frac{1}{N-N_{1}} \sum_{k=N_{1}}^{N} \sum_{t=1}^{n}\left[y_{k}(t)-\hat{y}_{k}(t)\right]^{2} \leq \varepsilon_{2} .
$$

In the actual application process, $E_{1}$ is often very small, but $E_{2}$ cannot meet the requirements. This is the so-called "over-fitting" phenomenon. Usually, only the BP neural network whose $E_{1}$ and $E_{2}$ meet the requirements has the practical value.

\section{GM-BPANN Improved Algorithm Flow}

As the core of the subject of artificial intelligence, neural networks are the basis for making computers intelligent. They are interoperable and inclusive and can organically integrate multiple types of theories. The main content is to use algorithms to enable the machine to have a certain simulation ability, to independently complete some behaviors that only humans can do before, to establish the machine's own knowledge structure and skill system, and to update and improve its knowledge structure in real time. As one of the most important technologies of artificial intelligence, it is the basis for the realization of mechanical 


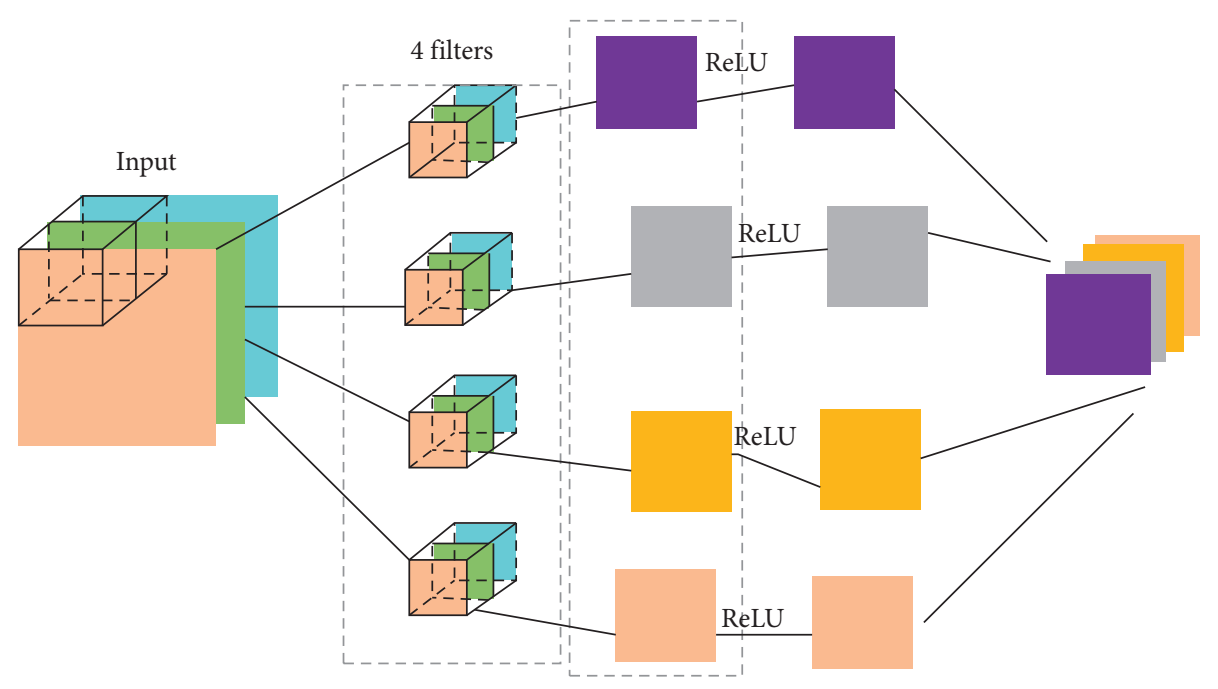

Figure 2: BP method sketch.

intelligence. Generally speaking, neural networks are mainly used to summarize and integrate information, which is then deduced by artificial intelligence.

When we study a multivariate complex system, in order to avoid the omission of factors that have an important impact on the dependent variable, the most common method is that using a qualitative analysis method to select a large number of independent variables that have a greater impact on the dependent variable and then start to build a system model. When these factors (independent variables) are many, using them as the input of the GA-BPANN (Figure 3) will have a large number of input variables can be processed, and the variables selected by it can be as comprehensive and extensive as possible, without special subjective screening. In this way, we first use the gray correlation analysis to filter out the variables (independent variables) that have the most important impact on the dependent variable (network output) as the input nodes and then use the BP algorithm for learning, thereby, enhancing the BP algorithm's multivariable complex system modeling capabilities. The algorithm flow is shown in Figure 4.

\section{Application of the GM-BPANN Model Based on Gray Relational Analysis in National Grain Output Forecast}

5.1. Selection and Processing of Predictors. In addition to uncertain factors, the factors affecting grain production are usually divided into three categories: natural environment, meteorology, and socioeconomic factors. According to the two principles that can be measured and have agronomic significance and combined with the results of preliminary influencing factor analysis, this study selects the total grain output $(Y)$ from 1978 to 2004 as the output factor and initially selects the sown area of grain crops $\left(x_{1}\right)$ and chemical fertilizers in the same time period. Application rate $\left(x_{2}\right)$, effective irrigation area of food crops $\left(x_{3}\right)$, disaster area $\left(x_{4}\right)$, rural electricity consumption $\left(x_{5}\right)$, total power of agricultural machinery $\left(x_{6}\right)$, primary industry employees $\left(x_{7}\right)$,

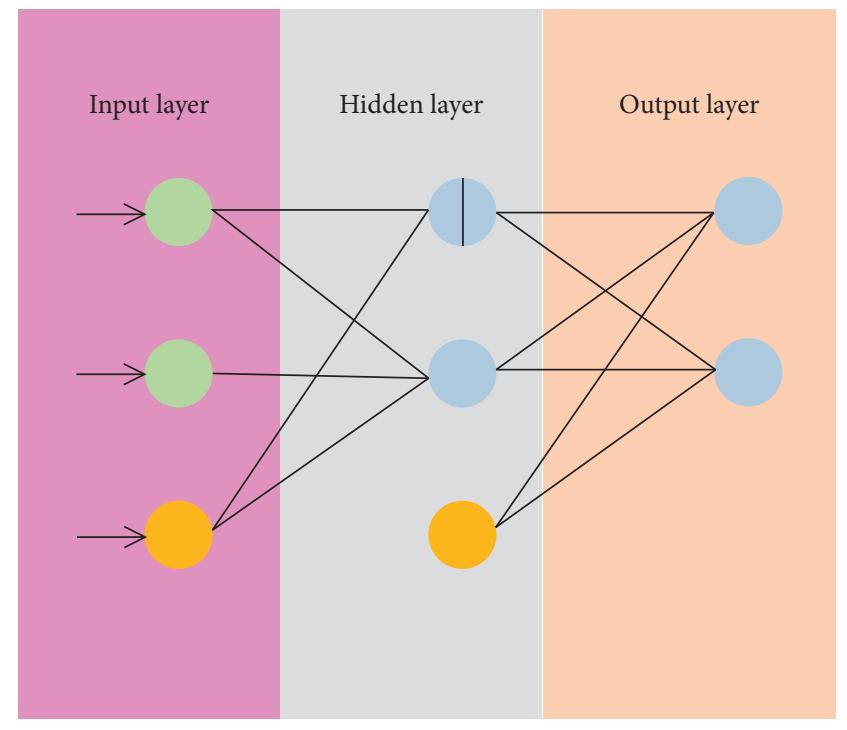

FIGURE 3: Reverse neural network.

capital construction expenditure $\left(x_{8}\right)$, and agricultural science and technology 12 factors including cost $\left(x_{9}\right)$, total agricultural expenditure $\left(x_{10}\right)$, average net income of rural households $\left(x_{11}\right)$, and average net income of rural households from business operations $\left(x_{12}\right)$ are used as input factors to construct the model.

With the aim of eliminating the influence of dimensions, the $Z$-score transformation method is used to transform the collected sample data, that is, the mean value and standard deviation $S$ of the variable data are first obtained, and then, the mean value of the variable is subtracted from the variable value and then divided by the standard deviation of the variable, that is, the standardized value is obtained, and the formula is expressed as follows: $X^{\prime}=(X-\bar{X}) / S$ uses DPS software to perform gray correlation analysis on the data from 1978 to 2004, and the results are as follows.

Using the results of the above gray correlation analysis, we set the training parameters of the BP network as follows: the number of iteration steps $M_{0}=5000$, the error threshold 


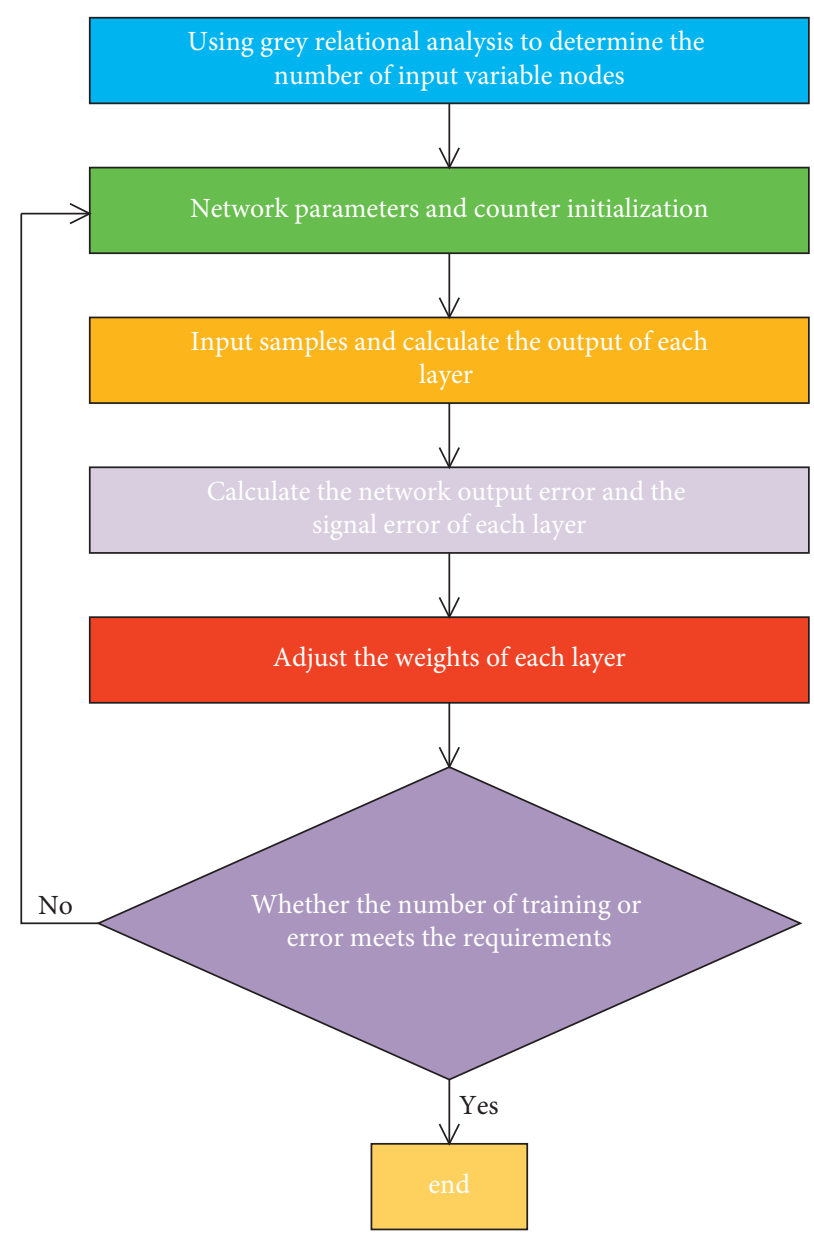

Figure 4: Algorithm flow.

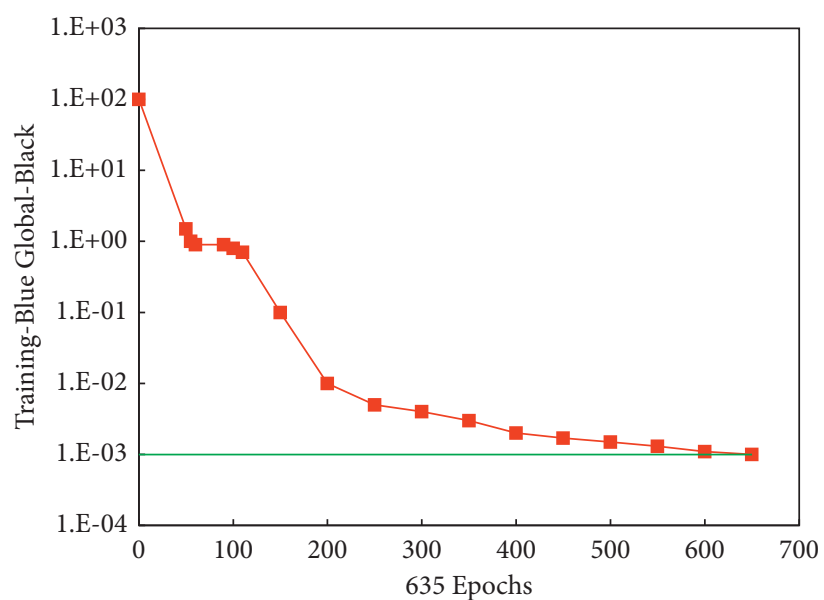

FiguRe 5: Network training.

$\varepsilon=0.001$, the learning parameter $b=0.5$, and the momentum term coefficient $a=0.1$. The training samples are 21 samples from 1978 to 1999 , and the test samples are 5 independent samples from 2000 to 2004. Using the Matlab neural network toolbox (nnet) to learn and filter the algorithm, get the optimal BP neural network structure 7-5-1 (input layer node 2 hidden layer node 2 output layer node).
Use the training sample data to build the prediction model. The network training process and error curve are shown in Figures 5 and 6 . As can be seen, a good agreement can be obtained, and it also shows the validation of the proposed method. It is found from Figure 4 that under the selected parameters, after more than 600 iterations, the model converges, the convergence performance is better, 


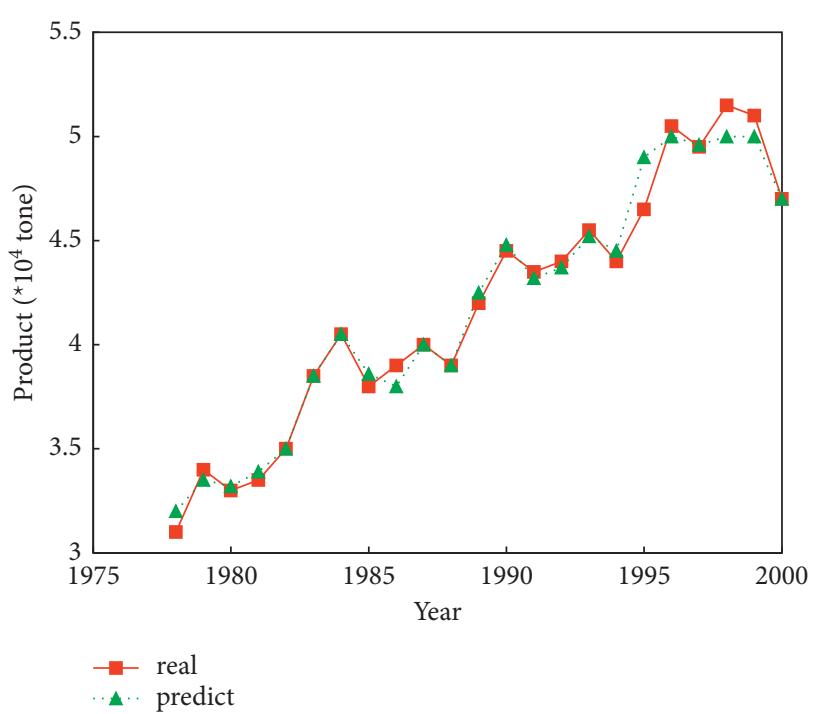

Figure 6: Error curve.

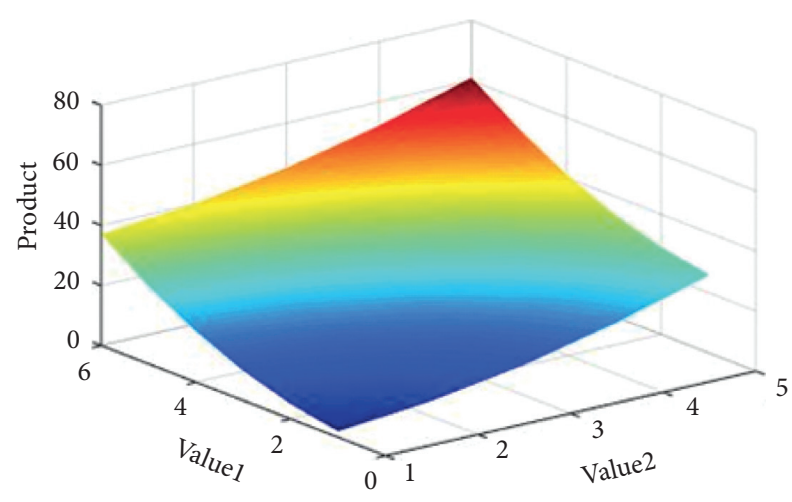

FIgURE 7: Regression prediction.

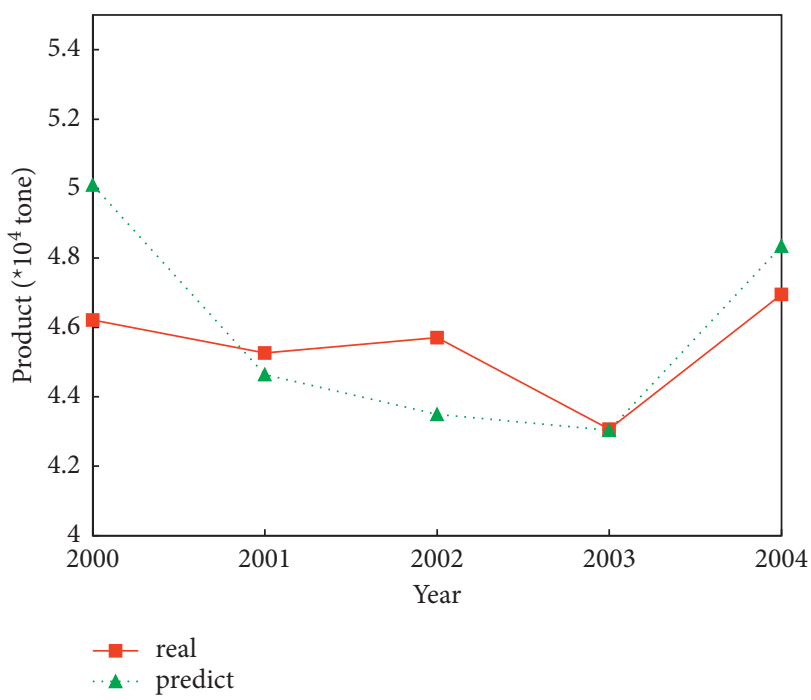

Figure 8: GM $(1, N)$ prediction.

and it does not fall into a local minimum. Besides, the predictions and real data from 2000 to 2004 are also compared in Figures 7-9 made by Matlab.

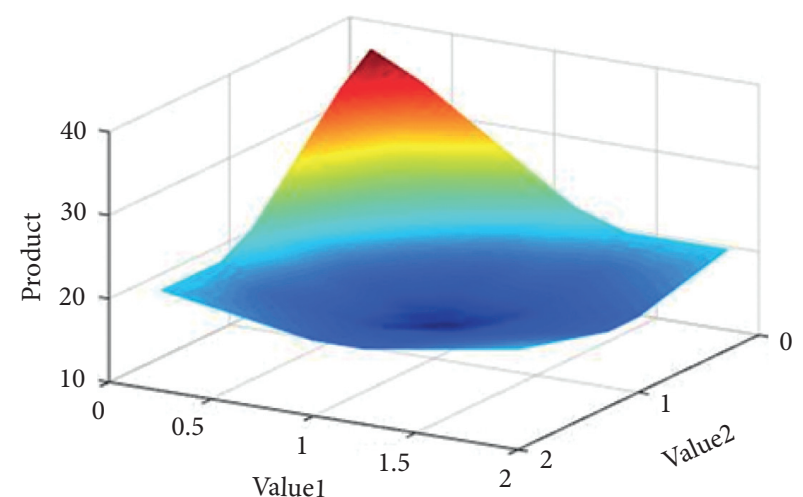

Figure 9: GM-BPANN prediction.

\section{Conclusions}

The results show that the new model can comprehensively and extensively process a large number of input variables by using the concept of the gray correlation degree, without having to go through special subjective screening and hence improving the adaptability of the BP network, and at the same time, it can obtain better prediction accuracy. Through the empirical test, the prediction ability of the three methods of the regression, GM $(1, N)$ gray system, and GM-BPANN model is compared. It is proved that the GM-BPANN optimization algorithm that combines the gray relational analysis and BP neural network method can enhance the multivariable processing ability and network adaptability of the BP network and has good prediction accuracy and stability.

Though the proposed BP neural network exhibits a good performance, it still needs to be improved since the convergence speed and training speed are still time consuming, and it is also the further work that is to be done in the future.

\section{Data Availability}

The data used to support the findings of this study are available from the corresponding author upon request.

\section{Conflicts of Interest}

The author declares that there are no conflicts of interest.

\section{Acknowledgments}

This study was supported by the MOE (Ministry of Education in China) Liberal Arts and Social Sciences Foundation, construction of an early warning model of social media users' mental health problems based on a deep learning framework (19YJCZH184).

\section{References}

[1] R. Z. Farahani, E. Miandoabchi, W. Y. Szeto, and H. Rashidi, "A review of urban transportation network design problems," European Journal of Operational Research, vol. 229, no. 2, pp. 281-302, 2013. 
[2] L. Xin, X. Li, Z. Zhang, and L. Zhao, "Seismic behavior of long-span concrete-filled steel tubular arch bridge subjected to near-fault fling-step motions," Engineering Structures, vol. 180, no. 1, pp. 148-159, 2019.

[3] J. Lv, T. Zhou, and K. Li, "Investigation and application of a new low-carbon material (preplaced aggregate concrete) in concrete-filled steel tube stub columns," Sustainability, vol. 12, no. 5, pp. 1768-1779, 2020.

[4] J. Lv, T. Zhou, Q. Du, K. Li, and L. Jin, "Research on the bond behavior of preplaced aggregate concrete-filled steel tube columns," Materials, vol. 13, no. 2, pp. 300-313, 2020.

[5] J. Hou, W. Xu, Y. Chen, K. Zhang, H. Sun, and Y. Li, "Typical diseases of a long-span concrete-filled steel tubular arch bridge and their effects on vehicle-induced dynamic response," Frontiers of Structural and Civil Engineering, vol. 14, no. 4, pp. 867-887, 2020.

[6] A. M. El Hadidy, M. F. Hassanein, and M. Zhou, "The effect of using tubular flanges in bridge girders with corrugated steel webs on their shear behaviour-a numerical study," ThinWalled Structures, vol. 124, no. 1, pp. 121-135, 2018.

[7] W. Huang, L. Fenu, B. Chen, and B. Briseghella, "Experimental study on joint resistance and failure modes of concrete filled steel tubular (CFST) truss girders," Journal of Constructional Steel Research, vol. 141, no. 1, pp. 241-250, 2018.

[8] Z. Wang, H. Ren, Q. Shen, W. Sui, and X. Zhang, "Seismic performance evaluation of a steel tubular bridge pier in a fivespan continuous girder bridge system," Structures, vol. 31, no. 1, pp. 909-920, 2021.

[9] S. Clark and D. Watling, "Modelling network travel time reliability under stochastic demand," Transportation Research Part B: Methodological, vol. 39, no. 2, pp. 119-140, 2005.

[10] A. Sumalee, D. P. Watling, and S. Nakayama, "Reliable network design problem: case with uncertain demand and total travel time reliability," Transportation Research Record: Journal of the Transportation Research Board, vol. 1964, no. 1, pp. 81-90, 2006.

[11] S. Nakayama and J. Takayama, "Traffic network equilibrium model for uncertain demands," in Proceedings of the 82nd Transportation Research Board Annual Meeting, Washington, DC, USA, 2021.

[12] H. Shao, W. H. K. Lam, and M. L. Tam, "A reliability-based stochastic traffic assignment model for network with multiple user classes under uncertainty in demand," Networks and Spatial Economics, vol. 6, no. 3, pp. 173-204, 2019.

[13] Y. Sun and M. A. Turnquist, "Investment in transportation network capacity under uncertainty: simulated annealing approach," Transportation Research Record: Journal of the Transportation Research Board, vol. 2039, no. 1, pp. 67-74, 2007.

[14] K. An and H. K. Lo, "Two-phase stochastic program for transit network design under demand uncertainty," Transportation Research Part B: Methodological, vol. 84, pp. 157-181, 2016.

[15] A. Chen, J. Kim, S. Lee, and Y. Kim, "Stochastic multi-objective models for network design problem," Expert Systems with Applications, vol. 37, no. 2, pp. 1608-1619, 2020.

[16] H. Wang, W. H. K. Lam, X. Zhang, and H. Shao, "Sustainable transportation network design with stochastic demands and chance constraints," International Journal of Sustainable Transportation, vol. 9, no. 2, pp. 126-144, 2015.

[17] S.-M. Hosseininasab and S.-N. Shetab-Boushehri, "Integration of selecting and scheduling urban road construction projects as a time-dependent discrete network design problem," European Journal of Operational Research, vol. 246, no. 3, pp. 762-771, 2015.
[18] S.-M. Hosseininasab, S.-N. Shetab-Boushehri, S. R. Hejazi, and $\mathrm{H}$. Karimi, "A multi-objective integrated model for selecting, scheduling, and budgeting road construction projects," European Journal of Operational Research, vol. 271, no. 1, pp. 262-277, 2018.

[19] S. Ukkusuri and G. Patil, "Multi-period transportation network design under demand uncertainty," Transportation Research Part B: Methodological, vol. 43, no. 6, pp. 625-642, 2010.

[20] E. Shayanfar and P. Schonfeld, "Selecting and scheduling interrelated road projects with uncertain demand," Transportmetrica A: Transport Science, vol. 15, no. 2, pp. 1712-1733, 2019.

[21] N. Zhu, B. Jia, and C. Shao, "A robust optimization model of mixed transportation network design problem with uncertain OD demand," Logistics Technology, vol. 31, no. 5, pp. 63-66, 2012.

[22] M. T. Hagan, H. B. Demuth, and M. Beale, Neural Network Design, PWS Publishing Company, Boston, MA, USA, 2016.

[23] M. Dougherty, "A review of neural networks applied to transport," Transportation Research Part C: Emerging Technologies, vol. 3, no. 4, pp. 247-260, 2019.

[24] M. Patriksson and L. Wynter, "Stochastic mathematical programs with equilibrium constraints," Operations Research Letters, vol. 25, no. 4, pp. 159-167, 2019.

[25] A. Evgrafov and M. Patriksson, "On the existence of solutions to stochastic mathematical programs with equilibrium constraints," Journal of Optimization Theory and Applications, vol. 121, no. 1, pp. 65-76, 2004.

[26] P. Zhou and Z. Jiang, "Self-organizing map neural network ( $\mathrm{SOM}$ ) downscaling method to simulate daily precipitation in the Yangtze and Huaihe River Basin," Climatic and Environmental Research, vol. 21, no. 5, pp. 512-524, 2016.

[27] X. Xiao, "Analysis on the employment psychological problems and adjustment of retired athletes in the process of career transformation," Modern Vocational Education, vol. 5, no. 12, pp. 216-217, 2018.

[28] S. Sahoo and M. K. Jha, "Pattern recognition in lithology classification: modeling using neural networks, self-organizing maps and genetic algorithms," Hydrogeology Journal, vol. 25, no. 2, pp. 311-330, 2016.

[29] Y. Zhou and B. Yang, "Sports video athlete detection using convolutional neural network," Journal of Natural Science of Xiangtan University, vol. 39, no. 1, pp. 95-98, 2017.

[30] J. Pang, "Research on the evaluation model of sports training adaptation based on self-organizing neural network," Journal of Nanjing Institute of Physical Education (Natural Science Edition), vol. 16, no. 1, pp. 74-77, 2017.

[31] G. Querzola, C. Lovati, C. Mariani, and L. Pantoni, "A semiquantitative sport-specific assessment of recurrent traumatic brain injury: the TraQ questionnaire and its application in American football," Neurological Sciences, vol. 40, no. 9, pp. 1909-1915, 2019.

[32] J. Wang, X. Luo, and H. Yan, "Correlation analysis between injuries and functional movement screening for athletes of the national shooting team," Journal of Capital Institute of Physical Education, vol. 5, no. 4, pp. 352-355, 2016.

[33] G. Ma, "Research on the design of juvenile football players' sports injury prediction model," Automation Technology and Application, vol. 277, no. 7, pp. 141-144, 2018. 